\title{
Two-thirds of fixed partial prostheses (bridges) last 15 years
}

\section{Scurria M S, Bader J D, Shugars D A Meta-analysis of fixed partial denture survival: prosthesis and abutments. J \\ Prosthet Dent 1998; 79: 459-464}

Objective To assess survival rates for fixed partial dentures and their abutments.

Data sources Medline search 1966-1996 for studies reporting on the longevity of fixed partial dentures (FPD) (bridges, fixed partial dentures, crown \& bridgework, survival and longevity). References of identified articles were also searched. Only articles in English were included and 35 were identified.

Study selection Studies were included which contained data allowing calculation of annual survival, with a minimum follow-up period of 3 years, defined failures so as to allow classification, identified patient, provider and examiner status. The FPDs were predominantly conventional (less than $50 \%$ cantilever and less than $25 \%$ nonfull coverage retainers). Of the 35 initially identified only 8 matched these criteria.

Data extraction and synthesis Kaplan-Meier modelling was used to back-calculate annual survival probability and the studies combined using a meta-analytical Bayesian model to derive overall annual survival

Results Three categories of survival were derived. A narrow definition of failure i.e. loss or removal of the FPD, a broader definition i.e. including FPDs which had technically failed or needed replacement, and whether the abutment teeth had survived. Results are shown in the table below.

$\begin{array}{lll}\begin{array}{l}\text { FPD surviving } \\ \text { (failure }=\text { loss } \\ \text { or removal) }\end{array} & \begin{array}{l}\text { FPD surviving } \\ \text { (failure = loss or } \\ \text { removal + technical } \\ \text { failure of needing } \\ \text { replacement }\end{array} & \begin{array}{l}\text { of abutments } \\ \text { surviving }\end{array} \\ 92 \% & 87 \% & 96 \% \\ 75 \% & 69 \% & \text { N/A }\end{array}$

Conclusions Less than $15 \%$ of fixed partial dentures were removed or in need of replacement at 10 years, whereas one third were removed or in need of replacement at 15 years. Less than $5 \%$ of abutments were removed at 10 years.

Address M S Scurria, Assistant Professor, Department of Prosthodontics, School of Dentistry, University of North Carolina, Chapel Hill, North Carolina, USA

\section{Commentary}

In the past five years meta-analysis has been increasingly used in dental sciences. Today it has been recognised as a powerful statistical tool for inference of treatment results above subjective review papers. However, there are some drawbacks that evoked serious criticism. The most mentioned problems are that of the publication bias (there is a tendency to publish only positive results), the utility of 'academic results' for 'daily practice', and the more or less subjective in- and exclusion procedures performed by the investigators. An interesting discussion about the latter has been presented by Proskin and Volpe. ${ }^{1}$

The present study is adding new material into this discussion in the field of restorative dentistry. It describes a systematic review on the survival of fixed partial dentures. Comparison with a previously published meta-analysis of bridge survival, ${ }^{2}$ shows that the results were quite similar to each other even though different sets of studies were involved. The same phenomenon was seen in a comparison made to study the effect of different study sets in a metaanalysis on the caries inhibiting effect of dentifrices. ${ }^{1}$ Although meta-analyses are not true reproducible 'experiments', this analogy suggests that they seem to produce consistent results. The constancy might also indicate that on the scale of a meta-analysis, the quality of individual studies is of insignificant value. However, this is in contrast with proposals of experts in this field, in which it is stated that quality assessment of individual studies is necessary to describe the sample and indicate the reliability of the data. ${ }^{3}$ In this respect a meta-meta-analysis, in which the effects of in- and exclusion criteria are systematically reviewed, would be most welcome.

The present analysis is adding new information by including different categories of survival into the results. A distinction is made between 'technical' and 'biological' failures, which leads to two survival estimates: one of the restorations and one of the abutments carrying the restorations. Fortunately, the abutment survival appeared to be significantly better than the restorative survival, which means that if bridges fail, restoration is possible without extending the number of units involved in the majority of cases.

1 Proskin H M, Volpe A R. Meta-analysis in dental research: a paradigm for performance and interpretation. J Clin Dent 1994; 5: 19-26.

2 Creugers NH J, Käyser A F, van't Hof M A. A meta-analysis of durability data on conventional fixed bridges. Community Dent Oral Epidemiol 1994; 22: 448-452.

3 Antczak A A, Tang J, Chalmers T C. Quality assessment of randomized control trials in dental research (II). Results: periodontal research.J Periodont Res 1986; 21: 315-321.

\section{N Creugars}

Professor of Restorative Dentistry, University of Nijmegen, The Netherlands 